\title{
Reproductive modes and fecundity of an assemblage of anuran amphibians in the Atlantic rainforest, Brazil
}

\author{
Marilia T. Hartmann ${ }^{1}$, Paulo A. Hartmann ${ }^{1}$ \& Célio F. B. Haddad ${ }^{2}$ \\ 1. Universidade Federal da Fronteira Sul, Campus Erechim, Avenida Dom João Hoffman, 313, 99700-000 Erechim, Rio Grande do Sul, \\ Brazil. (marilia.hartmann@gmail.com; hartmann.paulo@gmail.com) \\ 2. Departamento de Zoologia, Instituto de Biociências, Universidade Estadual Paulista, Caixa Postal 199, 13506-900 Rio Claro, São \\ Paulo, Brazil. (haddad1000@gmail.com)
}

\begin{abstract}
Reproductive modes and size-fecundity relationships are described for anurans from Picinguaba, a region of Atlantic rainforest on the northern coast of the state of São Paulo, Brazil. We observed 13 reproductive modes, confirming a high diversity of modes in the Atlantic rainforest. This diversity of reproductive modes reflects the successful use of diversified and humid microhabitats by anurans in this biome. We measured the snout-vent length of 715 specimens of 40 species of anurans. The size-fecundity relationship of 12 species was analyzed. Female snout-vent lengths explained between $57 \%$ and $81 \%$ of clutch size variation. Anurans with aquatic modes laid more eggs than those with terrestrial or arboreal modes. Larger eggs were deposited by species with specialized reproductive modes.
\end{abstract}

KEYWORDS. Reproduction, Anura, clutch size, community.

RESUMO. Modos reprodutivos e fecundidade de anfíbios anuros em uma taxocenose na Mata Atlântica, Brasil. Neste estudo são apresentados os modos reprodutivos e as relações de tamanho-fecundidade dos anuros encontrados em Picinguaba, uma região de Mata Atlântica no litoral norte do estado de São Paulo. Foram registrados 13 modos reprodutivos, confirmando a alta diversidade de modos reprodutivos encontrados na Mata Atlântica. A diversidade de modos reprodutivos observados na Mata Atlântica é o resultado do sucesso dos anuros na utilização dos diversos microhábitats úmidos desse bioma. Os comprimentos rostro-cloacais de 715 indivíduos de 40 espécies de anuros foram medidos. Foram consideradas as relações de tamanho-fecundidade para 12 espécies. O tamanho do corpo explicou entre $57 \%$ e $81 \%$ da variação no tamanho da ninhada. Os modos reprodutivos aquáticos apresentaram maior número de ovos, comparados aos modos reprodutivos terrestres ou arborícolas. As espécies com maior tamanho de ovos foram as que apresentaram modos reprodutivos especializados.

PALAVRAS-CHAVE. Reprodução, Anura, tamanho da ninhada, comunidade.

Reproductive mode is defined as a combination of oviposition factors and development, including oviposition site, egg and clutch characteristics, rate and duration of development, stage and size of hatchling and type of parental care, when it occurs (Duellman \& TrueB, 1986). Amongst vertebrates, amphibians show the greatest diversity of reproductive modes (CALDwELL, 1992). Duellman (1985) recognized 29 reproductive modes for anurans, of which 21 were found in the neotropics and eight were endemic to this region (CALDWELL, 1992). Recently, Haddad \& Prado (2005) reviewed the reproductive modes in anurans and recognized 39 reproductive modes divided into three large categories: aquatic eggs, terrestrial or arboreal eggs, and eggs carried by adults or retained in oviducts. Of the 39 modes, 31 can be found in the Neotropical region. The reproductive modes of Hylidae from the Atlantic rainforest and of Leptodactylus Fitzinger, 1826 have also recently been reviewed (Haddad \& SAWAYa, 2000; Prado et al., 2002, respectively).

Reproductive modes vary from the most generalized modes, with aquatic eggs and larvae, to the most specialized, totally independent of water (CRUMP, 1982; Duellman, 1985; Duelman \& Trueb, 1986). Mainly in tropical regions, there seems to be a trend for eggs and tadpoles to be removed from aquatic environments, probably as a way of reducing predation in the first development stages (CALDWELL, 1992).
Within each reproductive mode, parameters such as body size, clutch size and egg diameter are similar among species (Salthe \& Duellman, 1973). These quantitative variables influence the maintenance of the genetic variability of the population (CRUMP, 1982).

Species richness also seems to be related with the diversity of reproductive modes (CRUMP, 1982), permitting the coexistence of a large number of species, mainly in tropical forests (SAlthe \& Duellman, 1973). Seasonal differences in reproduction and development sites are indicated as important characteristics for resource partitioning (Salthe \& Duellman, 1973). The partitioning of oviposition sites occurs as a function of the necessities of one particular reproductive mode (CRUMP, 1982).

The greater species richness and higher number of reproductive modes in forests is correlated with regions of high humidity (Lynch, 1979; Duellman \& ThOMAS, 1996), such as the Atlantic rainforest. Despite the great diversity of species and reproductive modes, the reproductive biology of the species of the Atlantic rainforest is still not well known. Some studies exist on reproductive biology at the community level (e.g. HEYER et al., 1990; Bertolluci, 1998; Bertolluci \& Rodrigues, 2002) and at population level (e.g. Carvalho, 1949; IzeCKSOHN \& JiM, 1971; Pombal et al., 1994; Martins et al., 1998; HadDAD \& GiaretTA, 1999; BoquimPani-Freitas et al., 2002; CARVALHO-E-SiLVA et al., 2002). 
In this study we present the reproductive modes and size-fecundity relationships of anurans from Picinguaba, a region of Atlantic rainforest in the northern coast of the state of São Paulo, Brazil.

\section{MATERIAL AND METHODS}

The study was carried out in the Parque Estadual da Serra do Mar, Núcleo Picinguaba, Ubatuba (23⒉ $23^{\prime} \mathrm{S}$, $44^{\circ} 50^{\prime}$ W), state of São Paulo, Brazil, from January 2000 to November 2002. Rainfall is high throughout the year, with a mean annual precipitation of 2000 to $2500 \mathrm{~mm}$. The warmest and rainiest months are January, February and March.

The study was carried out at sea level and at altitudes up to $200 \mathrm{~m}$, in three defined vegetation types (the study area has seven vegetation types, see VELOSO et al., 1991): (1) dense ombrophilous forest (Atlantic rainforest), (2) restinga (beach vegetation, dunes, coastal forests and caxetais), and (3) transitional vegetation between the Atlantic rainforest and the restinga and fertile valley (swamps to temporarily flooded terraces).

Observations were made during the day and at night. For each species found in reproductive activity the following characteristics were recorded: (1) calling site, (2) presence of amplectant pairs or courtship behavior, (3) site of courtship or amplexus, and (4) site and shape of spawn. Reproductive behavior, from pair formation to oviposition, was monitored whenever possible. Amplectant pairs were collected and kept in plastic bags with a film of water in order to collect spawn. The modes of Rhinella icterica (Spix, 1824), Haddadus binotatus (Spix, 1824), Ischnocnema bolbodactyla (A. Lutz, 1925), I. guentheri (Steindachner, 1864), I. parva (Girard, 1853), Arcovomer passarellii Carvalho, 1954, and Myersiella microps (Duméril \& Bibron, 1841) were not observed in the field and were inferred from the literature or from observations of the calling sites.

Gravid females, found in nature, were either fixed in formalin and dissected by a ventral incision or placed in plastic bags with conspecific males in order to obtain spawn. The ovules obtained from dissecting the females and from the spawn were weighed with a digital scale of $0.01 \mathrm{~g}$ precision. The total number of eggs was counted in clutches and ovarian complements of up to 500 eggs and was estimated from total mass in larger clutches. The diameter of 10 eggs per clutch was measured using the stereomicroscope, with a micrometric ocular lens.

For the observation of tadpoles, some of the clutches were kept in aquaria; in the field, tadpoles were collected with a fish net. Tadpoles were fixed in formaldehyde $5 \%$, for comparison with the literature and/ or material from scientific collections. Some tadpoles were reared to metamorphosis for identification.

All material collected was deposited in the Célio F. B. Haddad collection, Departamento de Zoologia, Instituto de Biociências, Universidade Estadual Paulista, Rio Claro, São Paulo, Brazil. Collection permits were issued by the Instituto Brasileiro do Meio Ambiente e dos Recursos Naturais Renováveis, license number 025/ 02 - RAN - IBAMA.

The lentic water bodies were classified as: temporary ponds-lasting from two days to one week; semi-temporary ponds-lasting from one to two months; semi-permanent ponds-lasting the entire rainy period. In Picinguaba, only sites with running water were considered permanent. Nomenclature of the studied species follows Frost (2010); reproductive modes follows HADDAD \& PRADO (2005); type of oviposicional modes follows Altig \& MCDiarmids (2007); description of reproductive mode of Aplastodiscus eugenioi (Carvalhoe-Silva \& Carvalho-e-Silva, 2005) follows HARTMANN et al. (2004); description of reproductive mode of Physalaemus atlanticus Haddad \& Sazima, 2004 follows HADDAD \& SAZIMA (2004).

Linear simple regression analyses were used to test the relationship between size of female (snout-vent length) and size of clutch (number of ovules counted in each female), and size of clutch and mass of female. Sexual differences in body size (snout-vent length) were tested with the Student t-test.

\section{RESULTS}

Thirteen reproductive modes were observed for 40 species of anurans in Picinguaba. A total of 27 species deposited their eggs in the water (reproductive modes related with aquatic eggs). The majority of these species $(n=17)$ deposited eggs in ponds formed by rainwater (temporary, semi-temporary or semi-permanent). The largest ponds formed by rainwater in Picinguaba were located in open areas and dried out during three to four months of the year, when there was less rainfall. When these ponds were full, up to 12 species of anurans were observed calling syntopically in the same night.

The reproductive modes for Picinguaba are described below, with commentaries on the observed species.

Aquatic eggs. Mode 1. Eggs and exotrophic tadpoles in lentic water: Rhinella ornata (Spix, 1824), Macrogenioglottus alipioi Carvalho, 1946, Dendropsophus elegans (Wied-Neuwied, 1824), D. giesleri (Mertens, 1950), D. minutus (Peters, 1872), Hypsiboas albomarginatus (Spix, 1824), H. faber (WiedNeuwied, 1821), Itapotihyla langsdorffii (Duméril \& Bibron, 1841), Scinax alter (B. Lutz, 1973), S. angrensis (B. Lutz, 1973), S. argyreornatus (Miranda-Ribeiro, 1926), S. eurydice (Bokermann, 1968), S. hayii (Barbour, 1909), Trachycephalus mesophaeus (Hensel, 1867), Arcovomer passarellii, Chiasmocleis atlantica Cruz, Caramaschi \& Izecksohn, 1997, and C. carvalhoi Cruz, Caramaschi \& Izecksohn, 1997.

Explosive breeders (sensu WELLS, 1977) with mode 1 such as Itapotihyla langsdorffii, Scinax angrensis, S. argireornatus, Trachycephalus mesophaeus, Chiasmocleis carvalhoi and Arcovomer passarelli used mainly ponds at the forest edge or in the forest interior (the latter species reproduced only in temporary ponds in the forest interior). Tadpoles of these species were observed at different development stages in lentic waters of temporary ponds.

Although all species with mode 1 deposit their eggs in lentic water, differences in the type of oviposicional modes were observed. Rhinella ornata deposited eggs in a long thin chain of jelly, whilst in other species the eggs were deposited inside an individual gelatinous 
capsule. Depending on the ovipositon behavior, the spawn was either found spread over the water or divided in several portions on the water. In T. mesophaeus, the eggs were deposited slowly and the amplectant pair was observed swimming and depositing their eggs over a large area of the pond. The egg clutches of several pairs mixed together forming a large gelatinous mass surrounding water plants that emerged in the temporary ponds (oviposicional mode: film). In C. carvalhoi, the pairs were observed swimming around the water plants while they deposited their eggs, which got caught on the vegetation (oviposicional mode: film). In I. langsdorffii, the amplectant pair remained stationary until all the eggs were deposited and floated on the water surface (oviposicional mode: film). The clutches of species that used lentic water bodies had a high number of eggs which were small and pigmented (Tab. I).

Mode 2. Eggs and exotrophic tadpoles in lotic water: Rhinella icterica, R. ornata, Proceratophrys appendiculata (Günther, 1873), Hypsiboas semilineatus (Spix, 1824), and Scinax trapicheiroi (B. Lutz, 1954).

Rivulets and small streams served as breeding sites for species that develop in running waters. In the lotic waters, spawns were observed mainly in stream backwaters. Hypsiboas semilineatus and $P$. appendiculata bred exclusively in lotic environments.

Mode 3. Eggs and early larval stages in constructed subaquatic chambers; exotrophic tadpoles in streams: Hylodes asper (Müller, 1924) and H. phyllodes Heyer \& Cocroft, 1986. These two species call from emergent rocks in streams and deposit their eggs in subaquatic chambers. After hatching, we observed the tadpoles in the running waters.

Mode 4. Eggs and early larval stages in natural or constructed basins; subsequent to flooding, exotrophic tadpoles in ponds or streams: Bokermannohyla sp. (aff. circumdata) and Hypsiboas faber.

Bokermannohyla sp. (aff. circumdata) used water bodies for oviposition that ranged from rainwater puddles on the roadside to rapid streams. The clutches of this species contain large eggs protected by large jelly capsules and were found in small natural or constructed basins (Ivan Sazima, pers. comm.) nearby streams or beside forest trails. The reproduction of Hypsiboas faber was characterized by the construction of mud-basin nests on the bank of semi-permanent ponds, from which the males call. In some cases, the male was observed calling from the pond edge and not from inside the basin. The eggs of $H$. faber are numerous, small and dark, and are observed floating on the surface of the water contained inside the mud-basins constructed by the males (oviposicional modes: film).

Mode 5. Eggs and early larval stages in subterranean constructed nests; subsequent to flooding, exotrophic tadpoles in ponds or streams: Aplastodiscus eugenioi. This species constructs an underground nest, with a surface opening, in muddy areas inside the forest. The males called from bromeliads or leaves close to the burrow. The eggs of this species are deposited inside the subterranean nest, floating on permeated water (film), and are not pigmented.
Mode 8. Eggs and endotrophic tadpoles in water in tree holes or aerial plants: Dendrophryniscus brevipollicatus Jiménez de la Espada, 1871. The clutch containing discolored eggs was deposited in water accumulated in epiphytic bromeliads. Adults and tadpoles were also found in this accumulated water.

Eggs in foam nest (aquatic). Mode 11. Foam nest floating on pond; exotrophic tadpoles in ponds: Leptodactylus latrans (Steffen, 1815) and Physalaemus atlanticus. Large foam nests were deposited by L. latrans either on the edge or on the water of semi-temporary or semi-permanent ponds. $P$. atlanticus deposited eggs in foam nests on the water surface and anchored to plants (mode 11) or in small foam nests in muddy areas amongst the leaf litter or directly on the forest floor (mode 28), a terrestrial reproductive mode described below.

Terrestrial or arboreal eggs. Eggs on ground, on rocks, or in borrows. Mode 19. Eggs on humid rocks or in rock crevices in a water film, exotrophic semiterrestrial tadpoles in the same environments: Cycloramphus boraceiensis Heyer, 1983 and Thoropa taophora (Miranda-Ribeiro, 1923). Both species are typical streamdwellers and their semiterrestrial eggs and tadpoles live on rocks where tiny rivulets flow. The tadpoles remain on the humid rocks until metamorphosis. Thoropa taophora was also found on humid rocky shores, where a water film flows over the rocks, giving a suitable environment for the development of the tadpoles.

Mode 23. Eggs with direct development deposited on the ground: Brachycephalus hermogenesi (Giaretta \& Sawaya, 1998), Haddadus binotatus, Ischnocnema bolbodactyla, I. guentheri, I. parva, and Myersiella microps. The dissected females of these species had a small number of large eggs (Tab. I), all unpigmented. Males call from the leaf litter, not associated with any water body.

Arboreal eggs. Mode 24. Eggs deposited on leaves hanging over lentic water bodies; eggs hatching into exotrophic tadpoles that drop into lentic water: Dendropsophus berthalutzae (Bokermann, 1962). The males of this species were heard calling during and after rainfall. The tadpoles were observed in lentic waters.

Mode 25. Eggs deposited on leaves hanging over lotic water bodies; eggs hatching into exotrophic tadpoles that drop into lotic water: Vitreorana uranoscopa (Müller, 1924), and Phasmahyla guttata (A. Lutz, 1924). The spawn of $P$. guttata is hidden inside a leaf with edges folded into a type of envelope and in V. uranoscopa the spawn is exposed on the leaf. The tadpoles of $P$. guttata form schools in pools formed in fast-flowing streams, where the water is continuously renewed. Tadpoles were found in the same locality during 10 consecutive months. The tadpoles of $V$. uranoscopa are unpigmented with vestigial eyes and fossorial habits.

Eggs in foam nest (terrestrial or arboreal). Mode 28. Foam nest on the humid forest floor; exotrophic tadpoles in lentic water bodies: Physalaemus atlanticus. In this species the eggs were observed in a foam nest deposited on the water surface and anchored to plants (mode 11), or alternatively, on the leaf litter nearby ponds (mode 28). The foam nests on the ground are taken to the temporary ponds subsequent to flooding. 
Table I. Biometric and fecundity data for anurans species analyzed at Picinguaba, Ubatuba, state of São Paulo, Brazil from January 2000 to November 2002 (*, clutches deposited by amplectant pairs and maintained in plastic bags; **, tests with statistically significant results; SVL, snout-vent length).

\begin{tabular}{|c|c|c|c|c|c|c|c|c|c|}
\hline \multirow[b]{2}{*}{ Family/Species } & \multicolumn{2}{|l|}{$\begin{array}{l}\text { SVL } \\
\text { males }\end{array}$} & \multicolumn{2}{|l|}{$\begin{array}{c}\text { SVL } \\
\text { females }\end{array}$} & \multirow{2}{*}{$\begin{array}{l}\text { Sexual } \\
\text { dimorphism } \\
t \text { test }\end{array}$} & \multicolumn{2}{|l|}{$\begin{array}{l}\text { Number } \\
\text { of eggs }\end{array}$} & \multicolumn{2}{|l|}{$\begin{array}{l}\text { Diameter } \\
\text { of eggs }\end{array}$} \\
\hline & $\begin{array}{l}\text { Mean } \pm \text { SD } \\
\text { (range) }\end{array}$ & $\mathrm{n}$ & $\begin{array}{l}\text { Mean } \pm \text { SD } \\
\text { (range) }\end{array}$ & $\mathrm{n}$ & & $\begin{array}{l}\text { Mean } \pm \text { SD } \\
\text { (range) }\end{array}$ & $\mathrm{n}$ & $\begin{array}{l}\text { Mean } \pm \text { SD } \\
\text { (range) }\end{array}$ & $\mathrm{n}$ \\
\hline $\begin{array}{l}\text { BRACHYCEPHALIDAE } \\
\text { Brachycephalus hermogenesi } \\
\text { (Giaretta \& Sawaya, 1998) }\end{array}$ & $\begin{array}{l}9.37 \pm 0.01 \\
(9.36-9.38)\end{array}$ & 2 & & & & & & & \\
\hline $\begin{array}{l}\text { Haddadus binotatus } \\
\text { (Spix, 1824) }\end{array}$ & $\begin{array}{l}41.37 \pm 6.69 \\
(31.64-51.02)\end{array}$ & 11 & $\begin{array}{l}56.51 \pm 3.50 \\
(50.30-60.94)\end{array}$ & 6 & $\begin{array}{l}* * t=5.11 \\
\mathrm{p}=0.00\end{array}$ & $\begin{array}{l}31.75 \pm 1.50 \\
(30-33)\end{array}$ & 4 & $\begin{array}{l}3.59 \pm 1.07 \\
(1.90-5.00)\end{array}$ & 16 \\
\hline $\begin{array}{l}\text { Ischnocnema bolbodactyla } \\
\text { (A. Lutz, 1925) }\end{array}$ & $\begin{array}{l}14.71 \pm 0.54 \\
(13.38-15.32)\end{array}$ & 13 & $(18.87)$ & 1 & $\begin{array}{l}* * \mathrm{t}=7.48 \\
\mathrm{p}=0.00\end{array}$ & & & & \\
\hline $\begin{array}{l}\text { I. guentheri } \\
\text { (Steindachner, 1864) }\end{array}$ & $\begin{array}{l}24.30 \pm 2.44 \\
(20.74-27.32)\end{array}$ & 5 & (33.58) & 1 & $\begin{array}{l}* * \mathrm{t}=3.46 \\
\mathrm{p}=0.02\end{array}$ & (27) & 1 & & \\
\hline $\begin{array}{l}\text { I. parva } \\
\text { (Girard, 1853) } \\
\text { BUFONIDAE }\end{array}$ & $\begin{array}{l}15.53 \pm 1.63 \\
(13.68-18.90)\end{array}$ & 11 & $\begin{array}{l}17.58 \pm 2.02 \\
(13.39-19.85)\end{array}$ & 8 & $\begin{array}{l}* * \mathrm{t}=2.45 \\
\mathrm{p}=0.02\end{array}$ & $\begin{array}{l}11 \pm 3.16 \\
(8-15)\end{array}$ & 4 & $\begin{array}{l}4.05 \pm 0.26 \\
(3.70-4.30)\end{array}$ & 4 \\
\hline $\begin{array}{l}\text { Rhinella ornata } \\
\text { (Spix, 1824) }\end{array}$ & $\begin{array}{l}61.43 \pm 3.66 \\
(54.32-9.58)\end{array}$ & 18 & $\begin{array}{l}73.37 \pm 6.82 \\
(59.41-83.87)\end{array}$ & 12 & $\begin{array}{l}* * \mathrm{t}=6.22 \\
\mathrm{p}<0.00\end{array}$ & $\begin{array}{l}4222.25 \pm 1614.71 \\
(1477-5964) \\
(* 2589)\end{array}$ & $\begin{array}{l}18 \\
1\end{array}$ & $\begin{array}{l}1.4 \pm 0.17 \\
(0.95-1.71)\end{array}$ & 16 \\
\hline $\begin{array}{l}\text { R. icterica } \\
\text { (Spix, 1824) }\end{array}$ & (118.7) & 1 & & & & & & & \\
\hline $\begin{array}{l}\text { Dendrophryniscus brevipollicatus } \\
\text { Jiménez de la Espada, } 1871 \\
\text { CENTROLENIDAE }\end{array}$ & $\begin{array}{l}15.90 \pm 1.12 \\
(14.58-17.57)\end{array}$ & 6 & $\begin{array}{l}19.72 \pm 2.01 \\
(17.19-22.80)\end{array}$ & 8 & $\begin{array}{l}* * \mathrm{t}=4.12 \\
\mathrm{p}<0.00\end{array}$ & $\begin{array}{l}18.50 \pm 9.00 \\
(9-32)\end{array}$ & 6 & $\begin{array}{l}1.59 \pm 0.42 \\
(0.95-2.30)\end{array}$ & 17 \\
\hline $\begin{array}{l}\text { Vitreorana uranoscopa } \\
\text { (Müller, 1924) } \\
\text { CYCLORAMPHIDAE }\end{array}$ & $\begin{array}{l}20.09 \pm 1.25 \\
(18.41-21.45)\end{array}$ & 4 & & & & $(* 29)$ & 1 & $\begin{array}{l}2.03 \pm 0.08 \\
(1.90-2.20)\end{array}$ & 13 \\
\hline $\begin{array}{l}\text { Cycloramphus boraceiensis } \\
\text { Heyer, } 1983\end{array}$ & $\begin{array}{l}38.66 \pm 9.79 \\
(26.24-51.66)\end{array}$ & 10 & $\begin{array}{l}53.01 \pm 2.28 \\
(50.64-55.21)\end{array}$ & 4 & $\begin{array}{l}* * \mathrm{t}=2.83 \\
\mathrm{p}=0.01\end{array}$ & $\begin{array}{l}81.75 \pm 35.92 \\
(50-129)\end{array}$ & 4 & $\begin{array}{l}2.13 \pm 0.24 \\
(1.70-2.40)\end{array}$ & 8 \\
\hline $\begin{array}{l}\text { Macrogenioglottus alipioi } \\
\text { Carvalho, } 1946\end{array}$ & $\begin{array}{l}82.05 \pm 14.39 \\
(67.74-102.43)\end{array}$ & 7 & $\begin{array}{l}108.48 \pm 4.01 \\
(104.4-113.8)\end{array}$ & 4 & $\begin{array}{l}* * \mathrm{t}=3.52 \\
\mathrm{p}=0.00\end{array}$ & $(2900)$ & 1 & $\begin{array}{l}1.29 \pm 0.04 \\
(1.25-1.35)\end{array}$ & 4 \\
\hline $\begin{array}{l}\text { Proceratophrys appendiculata } \\
\text { (Günther, 1873) }\end{array}$ & $\begin{array}{l}45.34 \pm 2.68 \\
(42.52-47.86)\end{array}$ & 3 & $\begin{array}{l}51.24 \pm 8.26 \\
(45.40-57.09)\end{array}$ & 2 & $\begin{array}{l}\mathrm{t}=1.23 \\
\mathrm{p}=0.30\end{array}$ & $(355)$ & 1 & $\begin{array}{l}2.33 \pm 0.25 \\
(2-2.9)\end{array}$ & 12 \\
\hline $\begin{array}{l}\text { Thoropa taophora } \\
\text { (Miranda-Ribeiro, 1923) } \\
\text { HYLIDAE }\end{array}$ & $\begin{array}{l}72.75 \pm 9.58 \\
(57.57-83.66)\end{array}$ & 6 & $\begin{array}{l}72.43 \pm 1.59 \\
(70.04-73.28)\end{array}$ & 4 & $\begin{array}{l}\mathrm{t}=0.06 \\
\mathrm{p}=0.95\end{array}$ & $\begin{array}{l}765.66 \pm 450.49 \\
(345-1241)\end{array}$ & 3 & $\begin{array}{l}2.04 \pm 0.19 \\
(1.80-2.60)\end{array}$ & 20 \\
\hline $\begin{array}{l}\text { Aplastodiscus eugenioi } \\
\text { (Carvalho-e-Silva \& } \\
\text { Carvalho-e-Silva, 2005) }\end{array}$ & $\begin{array}{l}33.02 \pm 1.19 \\
(31.21-34.80)\end{array}$ & 14 & $\begin{array}{l}35.27 \pm 0.61 \\
(34.34-36.00)\end{array}$ & 6 & $\begin{array}{l}* * \mathrm{t}=0.27 \\
\mathrm{p}<0.00\end{array}$ & $\begin{array}{l}74.5 \pm 12.06 \\
(62-88)\end{array}$ & 5 & $\begin{array}{l}2.31 \pm 0.22 \\
(1.95-2.87)\end{array}$ & 56 \\
\hline $\begin{array}{l}\text { Bokermannohyla } \\
\text { aff. circumdata }\end{array}$ & $\begin{array}{l}63.06 \pm 3.92 \\
(59.89-68.80)\end{array}$ & 4 & $\begin{array}{l}62.64 \pm 3.79 \\
(65.89-66.17)\end{array}$ & 6 & $\begin{array}{l}\mathrm{t}=0.17 \\
\mathrm{p}=0.86\end{array}$ & $\begin{array}{l}342.5 \pm 98.28 \\
(273-412)\end{array}$ & 2 & & \\
\hline $\begin{array}{l}\text { Dendropsophus berthalutzae } \\
\text { (Bokermann, 1962) }\end{array}$ & $\begin{array}{l}18.91 \pm 1.44 \\
(14.80-20.60)\end{array}$ & 14 & $\begin{array}{l}22.10 \pm 1.07 \\
(21.34-22.86)\end{array}$ & 2 & $\begin{array}{l}* * \mathrm{t}=2.97 \\
\mathrm{p}=0.00\end{array}$ & $\begin{array}{l}12.6 \\
0)\end{array}$ & 6 & $\begin{array}{l}1.01 \pm 0.05 \\
(0.92-1.09)\end{array}$ & 11 \\
\hline $\begin{array}{l}\text { D. elegans } \\
\text { (Wied-Neuwied, 1824) }\end{array}$ & $\begin{array}{l}25.30 \pm 1.43 \\
(22.19-27.32)\end{array}$ & 13 & $\begin{array}{l}30.68 \pm 0.95 \\
(29.37-31.87)\end{array}$ & 5 & $\begin{array}{l}* * \mathrm{t}=768 \\
\mathrm{p}<<0.00\end{array}$ & $\begin{array}{l}* 375 \pm 42.57 \\
(328-411)\end{array}$ & 3 & $\begin{array}{l}1.26 \pm 0.07 \\
(1.15-1.40)\end{array}$ & 15 \\
\hline $\begin{array}{l}\text { D. giesleri } \\
\text { (Mertens, 1950) }\end{array}$ & $\begin{array}{l}25.16 \pm 1.54 \\
(23.55-28.65)\end{array}$ & 9 & $\begin{array}{l}34.52 \pm 1.93 \\
(33.16-35.89)\end{array}$ & 2 & $\begin{array}{l}* * \mathrm{t}=7.51 \\
\mathrm{p}<<0.00\end{array}$ & $\begin{array}{l}190 \pm 87.68 \\
(128-252)\end{array}$ & 2 & & \\
\hline $\begin{array}{l}\text { D. minutus } \\
\text { (Peters, 1872) }\end{array}$ & $\begin{array}{l}18.97 \pm 1.67 \\
(13.13-21.00)\end{array}$ & 23 & $\begin{array}{l}23.06 \pm 0.36 \\
(22.80-23.32)\end{array}$ & 2 & $\begin{array}{l}* * \mathrm{t}=3.37 \\
\mathrm{p}=0.00\end{array}$ & $\begin{array}{l}196.5 \pm 6.36 \\
(192-201)\end{array}$ & 2 & $\begin{array}{l}0.99 \pm 0.09 \\
(0.80-1.10)\end{array}$ & 10 \\
\hline $\begin{array}{l}\text { Hypsiboas albomarginatus } \\
\text { (Spix, 1824) }\end{array}$ & $\begin{array}{l}48.56 \pm 3.30 \\
(41.85-55.88)\end{array}$ & 18 & $\begin{array}{l}57.27 \pm 2.74 \\
(52.93-61.34)\end{array}$ & 12 & $\begin{array}{l}* * \mathrm{t}=7.54 \\
\mathrm{p}<<0.00\end{array}$ & $\begin{array}{l}1294.33 \pm 253.59 \\
(878-1617)\end{array}$ & 9 & & \\
\hline $\begin{array}{l}\text { H. faber } \\
\text { (Wied-Neuwied, 1821) }\end{array}$ & $\begin{array}{l}83.89 \pm 12.60 \\
(56.95-94.03)\end{array}$ & 7 & $(89.15)$ & 1 & $\begin{array}{l}\mathrm{t}=0.39 \\
\mathrm{p}=0.70\end{array}$ & $(1.724)$ & 1 & $\begin{array}{l}1.92 \pm 0.15 \\
(1.72-2.12)\end{array}$ & 8 \\
\hline $\begin{array}{l}\text { H. semilineatus } \\
\text { (Spix, 1824) }\end{array}$ & $\begin{array}{l}40.40 \pm 1.55 \\
(38.67-41.67)\end{array}$ & 3 & $(52.06)$ & 1 & $\begin{array}{l}* * \mathrm{t}=6.49 \\
\mathrm{p}=0.02\end{array}$ & (891) & 1 & $\begin{array}{l}1.06 \pm 0.13 \\
(0.95-1.25)\end{array}$ & 4 \\
\hline $\begin{array}{l}\text { Itapotihyla langsdorffii } \\
\text { (Duméril \& Bibron, 1841) }\end{array}$ & $\begin{array}{l}71.61 \pm 5.04 \\
(64.90-77.01)\end{array}$ & 9 & $\begin{array}{l}91.49 \pm 4.30 \\
(87.62-97.52)\end{array}$ & 4 & $\begin{array}{l}* * \mathrm{t}=6.82 \\
\mathrm{p}<<0.00\end{array}$ & $\begin{array}{l}1741.5 \pm 1457.3 \\
(711-2772) \\
(* 5214)\end{array}$ & $\begin{array}{r}2 \\
* 1\end{array}$ & $\begin{array}{l}1.2 \pm 0.22 \\
(0.98-1.55) \\
* 1.45 \pm 0.07 \\
(1.30-1.55)\end{array}$ & $\begin{array}{r}7 \\
* 13\end{array}$ \\
\hline $\begin{array}{l}\text { Phasmahyla guttata } \\
\text { (A. Lutz, 1924) }\end{array}$ & $\begin{array}{l}35.54 \pm 1.86 \\
(33.67-37.39)\end{array}$ & 3 & $(38.59)$ & 1 & $\begin{array}{l}\mathrm{t}=1.41 \\
\mathrm{p}=0.29\end{array}$ & $(40)$ & 1 & $\begin{array}{l}2.40 \pm 0.25 \\
(1.93-2.74)\end{array}$ & 10 \\
\hline $\begin{array}{l}\text { Scinax alter } \\
\text { (B. Lutz, 1973) }\end{array}$ & $\begin{array}{l}27.67 \pm 1.34 \\
(24.20-29.32)\end{array}$ & 14 & $\begin{array}{l}29.73 \pm 0.87 \\
(28.66-30.98)\end{array}$ & 7 & $\begin{array}{l}* * \mathrm{t}=3.67 \\
\mathrm{p}=0.00\end{array}$ & $\begin{array}{l}* 431.25 \pm 127.7 \\
(322-581)\end{array}$ & 4 & $\begin{array}{l}1.03 \pm 0.10 \\
(0.90-1.20)\end{array}$ & 10 \\
\hline $\begin{array}{l}\text { S. angrensis } \\
\text { (B. Lutz, 1973) }\end{array}$ & $\begin{array}{l}24.82 \pm 1.47 \\
(22.51-29.01)\end{array}$ & 26 & $\begin{array}{l}35.26 \pm 1.85 \\
(31.77-39.25)\end{array}$ & 22 & $\begin{array}{l}* * \mathrm{t}=21.69 \\
\mathrm{p}<<0.00\end{array}$ & $\begin{array}{l}743 \pm 295.6 \\
(305-1111) \\
* 442 \pm 103.9 \\
(369-516)\end{array}$ & $\begin{array}{r}5 \\
* 2\end{array}$ & $\begin{array}{l}1.04 \pm 0.16 \\
(0.79-1.46) \\
* 1.55 \pm 0.39 \\
(1.5-1.6)\end{array}$ & $* 16$ \\
\hline $\begin{array}{l}\text { S. argyreornatus } \\
\text { (Miranda-Ribeiro, 1926) }\end{array}$ & $\begin{array}{l}15.89 \pm 0.96 \\
(14.24-17.81)\end{array}$ & 17 & $\begin{array}{l}18.80 \pm 0.77 \\
(18.00-20.67)\end{array}$ & 15 & $\begin{array}{l}* * \mathrm{t}=3.67 \\
\mathrm{p}=0.00\end{array}$ & $\begin{array}{l}126.36 \pm 36.23 \\
(45-170)\end{array}$ & 11 & $\begin{array}{l}0.83 \pm 0.06 \\
(0.7-0.9)\end{array}$ & 16 \\
\hline
\end{tabular}




\begin{tabular}{|c|c|c|c|c|c|c|c|c|c|}
\hline $\begin{array}{l}\text { S. eurydice } \\
\text { (Bokermann, 1968) }\end{array}$ & $\begin{array}{l}41.45 \pm 2.89 \\
(37.45-45.02)\end{array}$ & 14 & $\begin{array}{l}43.13 \pm 1.09 \\
(42.09-44.27)\end{array}$ & 3 & $\begin{array}{l}t=0.96 \\
p=0.34\end{array}$ & $\begin{array}{l}* 1699 \pm 301.9 \\
(1486-1913)\end{array}$ & $* 2$ & & \\
\hline $\begin{array}{l}\text { S. hayii } \\
\text { (Barbour, 1909) }\end{array}$ & $\begin{array}{l}39.00 \pm 1.65 \\
(36.89-43.07)\end{array}$ & 15 & $\begin{array}{l}47.15 \pm 1.47 \\
(44.68-48.77)\end{array}$ & 7 & $\begin{array}{l}* * \mathrm{t}=11.11 \\
\mathrm{p}=0.00\end{array}$ & $\begin{array}{l}1587.16 \pm 494.14 \\
(1063-2308)\end{array}$ & 6 & $\begin{array}{l}1.45 \pm 0.25 \\
(1.08-2.09)\end{array}$ & 17 \\
\hline $\begin{array}{l}\text { S. trapicheiroi } \\
\text { (B. Lutz, 1954) }\end{array}$ & $\begin{array}{l}26.15 \pm 0.89 \\
(24.09-27.5)\end{array}$ & 8 & & & & & & & \\
\hline $\begin{array}{l}\text { Trachycephalus mesophaeus } \\
\text { (Hensel, 1867) } \\
\text { HYLODIDAE }\end{array}$ & $\begin{array}{l}63.31 \pm 4.09 \\
(56.98-70.33)\end{array}$ & 11 & $\begin{array}{l}68.54 \pm 3.84 \\
(65.25-73.11)\end{array}$ & 4 & $\begin{array}{l}* * \mathrm{t}=2.21 \\
\mathrm{p}=0.04\end{array}$ & $\begin{array}{l}2367 \pm 488.08 \\
(1680-2781)\end{array}$ & 4 & $\begin{array}{l}1.5 \pm 0.17 \\
(1.20-1.90)\end{array}$ & 28 \\
\hline $\begin{array}{l}\text { Hylodes asper } \\
\text { (Müller, 1924) }\end{array}$ & $\begin{array}{l}29.64 \pm 5.98 \\
(24.16-38.42)\end{array}$ & 7 & $\begin{array}{l}41.38 \pm 3.93 \\
(35.18-44.89)\end{array}$ & 8 & $\begin{array}{l}* * \mathrm{t}=4.54 \\
\mathrm{p}=0.00\end{array}$ & $\begin{array}{l}81.4 \pm 26.21 \\
(57-114)\end{array}$ & 5 & $\begin{array}{l}3.14 \pm 0.47 \\
(2.5-4.00)\end{array}$ & 10 \\
\hline $\begin{array}{l}\text { H. phyllodes } \\
\text { Heyer \& Cocroft, } 1986 \\
\text { LEPTODACTYLIDAE }\end{array}$ & $\begin{array}{l}27.18 \pm 1.07 \\
(25.62-29.83)\end{array}$ & 19 & $\begin{array}{l}27.57 \pm 1.63 \\
(25.3-30.22)\end{array}$ & 6 & $\begin{array}{l}t=0.68 \\
p=0.50\end{array}$ & $\begin{array}{l}38.66 \pm 12.89 \\
(28-53)\end{array}$ & 3 & & \\
\hline $\begin{array}{l}\text { Leptodactylus marmoratus } \\
\text { (Steindachner, 1867) }\end{array}$ & $\begin{array}{l}20.17 \pm 1.12 \\
(17.9-22.9)\end{array}$ & 13 & $\begin{array}{l}22.02 \pm 0.92 \\
(19.29-23.05)\end{array}$ & 28 & $\begin{array}{l}* * \mathrm{t}=5.57 \\
\mathrm{p}=0.00\end{array}$ & $\begin{array}{l}5.15 \pm 2.52 \\
(2-10)\end{array}$ & 19 & $\begin{array}{l}3.02 \pm 1.15 \\
(1.55-5.50)\end{array}$ & 35 \\
\hline $\begin{array}{l}\text { L. latrans } \\
\text { (Steffen, 1815) } \\
\text { LEIUPERIDAE }\end{array}$ & $\begin{array}{l}98.65 \pm 13.23 \\
(77.86-121.25)\end{array}$ & 8 & $\begin{array}{l}86.96 \pm 9.40 \\
(80.36-100.82)\end{array}$ & 4 & $\begin{array}{l}\mathrm{t}=1.56 \\
\mathrm{p}=0.15\end{array}$ & $(5515)$ & 1 & $\begin{array}{l}0.56 \pm 0.08 \\
(0.50-0.70)\end{array}$ & 6 \\
\hline $\begin{array}{l}\text { Physalaemus atlanticus } \\
\text { Haddad \& Sazima, } 2004 \\
\text { MICROHYLIDAE }\end{array}$ & $\begin{array}{l}20.59 \pm 1.26 \\
(18.75-22.86)\end{array}$ & 17 & $\begin{array}{l}22.66 \pm 1.50 \\
(20.02-26.79)\end{array}$ & 17 & $\begin{array}{l}* * \mathrm{t}=4.33 \\
\mathrm{p}=0.00\end{array}$ & $\begin{array}{l}85.18 \pm 26.08 \\
(59-130)\end{array}$ & 11 & $\begin{array}{l}1.19 \pm 0.12 \\
(0.99-1.40)\end{array}$ & 13 \\
\hline $\begin{array}{l}\text { Arcovomer passarellii } \\
\text { Carvalho, } 1954\end{array}$ & & & $\begin{array}{l}22.36 \pm 0.84 \\
(21.39-22.93)\end{array}$ & 3 & & $\begin{array}{l}85.5 \pm 17.67 \\
(73-98)\end{array}$ & 2 & $\begin{array}{l}1.6 \pm 0.12 \\
(1.4-1.8)\end{array}$ & 11 \\
\hline $\begin{array}{l}\text { Chiasmocleis atlantica } \\
\text { Cruz, Caramaschi \& } \\
\text { Izecksohn, } 1997\end{array}$ & $(24.58)$ & 1 & $(31.56)$ & 1 & & $(730)$ & 1 & $\begin{array}{l}(1.13 \pm 0.16) \\
(0.82-1.31)\end{array}$ & 8 \\
\hline $\begin{array}{l}\text { C. carvalhoi } \\
\text { Cruz, Caramaschi \& } \\
\text { Izecksohn, } 1997\end{array}$ & $\begin{array}{l}18.07 \pm 0.89 \\
(16.24-20.31)\end{array}$ & 52 & $\begin{array}{l}22.77 \pm 1.17 \\
(20.05-24.41)\end{array}$ & 38 & $\begin{array}{l}* * \mathrm{t}=21.59 \\
\mathrm{p}=0.00\end{array}$ & $\begin{array}{l}236.83 \pm 69.08 \\
(123-395)\end{array}$ & 30 & $\begin{array}{l}1.21 \pm 0.10 \\
(0.94-1.37)\end{array}$ & 51 \\
\hline $\begin{array}{l}\text { Myersiella microps } \\
\text { (Duméril \& Bibron, 1841) }\end{array}$ & $\begin{array}{l}25.75 \pm 1.64 \\
(21.71-28.75)\end{array}$ & 14 & $\begin{array}{l}31.95 \pm 2.33 \\
(27.75-36.21)\end{array}$ & 10 & $\begin{array}{l}* * \mathrm{t}=7.66 \\
\mathrm{p}=0.00\end{array}$ & $\begin{array}{l}25.83 \pm 4.95 \\
(18-32)\end{array}$ & 6 & $\begin{array}{l}3.25 \pm 0.45 \\
(2.2-4)\end{array}$ & 16 \\
\hline Total & & 450 & & 265 & & & 181 & & 503 \\
\hline
\end{tabular}

Mode 32. Foam nest in subterranean constructed chambers; endotrophic tadpoles complete metamorphosis in nests: Leptodactylus marmoratus (Steindachner, 1867). The male of this species calls at the entrance of the burrow constructed in slopes or on the forest floor, where tadpoles remain until metamorphosis. Vocalizations were recorded during and after rainfall, at sunset, in almost the whole studied area.

Size-fecundity relationships. The snout-vent length (SVL) of 715 specimens from 40 species of anurans from eight families was measured (Tab. I). Small species were more common; 26 species had a SVL smaller than 50 mm (65\%; Tab. I). Most species showed statistically significant sexual size dimorphism ( $n=26$; Tab. I).

We counted 181 ovules from gravid females (Tab. I). Due to the small sample, size-fecundity relationships were only considered for 12 species with $n$ greater than four (Tab. II). Body size (SVL and mass) was only correlated with clutch size for three species: $R$. ornata, $P$. atlanticus and $C$. carvalhoi. Snout-vent length, rather than mass of body, explained the variation in clutch size (Tab. II). In the comparison between species, the number of eggs per clutch (using only data from ovarian oocytes) was positively correlated with the SVL of the female $\left(\mathrm{R}^{2}=0.54 ; \mathrm{p}=0.006 ; \mathrm{n}=12\right.$ species $)$. Aquatic reproductive modes were all above the trend line in the regression analysis (Fig. 1), showing that the species with aquatic eggs have a comparatively higher number of eggs per clutch relative to the SVL of the female.

Egg diameter was not statistically correlated with the size of the female $\left(\mathrm{R}^{2}=0.002 ; \mathrm{p}=0.90 ; \mathrm{n}=10\right.$ species;
Fig. 2). The biggest eggs belonged to M. microps and $L$. marmoratus, with terrestrial reproductive modes, and $A$. eugenioi and $H$. asper, with aquatic reproductive modes, albeit specialized. Physalaemus atlanticus, which presents two reproductive modes, one aquatic and one terrestrial, had an intermediate number and size of eggs between these two modes. The number of eggs per clutch in relation to the diameter of the eggs was statistically significant between the 12 species and had a negative relationship $\left(\mathrm{R}^{2}=0.42 ; \mathrm{p}=0.04\right)$.

When the analysis was separated by nonspecialized mode (only considering mode 1 ) and specialized mode (modes $8,5,3,32,28$, and 23 ), only considering the modes of the species in table II, a significant and positive relationship was found in the non-specialized reproductive mode between the number of eggs per clutch and the SVL of the female $\left(\mathrm{R}^{2}=0.96\right.$; $\mathrm{p}=0.0004 ; \mathrm{n}=6)$, but the relationship in the specialized reproductive modes was not statistically significant $\left(\mathrm{R}^{2}=\right.$ $0.34 ; \mathrm{p}=0.22 ; \mathrm{n}=6$ ). The relationship between diameter of egg and the size of the female was not statistically significant for either the non-specialized $\left(R^{2}=0.35 ; p=0.40\right.$; $\mathrm{n}=4)$ or the specialized $\left(\mathrm{R}^{2}=0.44 ; \mathrm{p}=0.14 ; \mathrm{n}=6\right)$ reproductive modes.

The mean number of eggs in species with terrestrial eggs (mean $=105.39 \pm 220.50, n=11$ species), was significantly lower $(\mathrm{t}=2.37, \mathrm{p}=0.02)$ than in species with aquatic eggs (mean $=1118.62 \pm 1394.52, \mathrm{n}=25$ species). The maximum diameter of eggs was greater in the species with terrestrial reproductive modes and smaller in the species with aquatic reproduction (Tab. I). 
Table II. Results of the linear regression analysis between snout-vent length of female (SVL) and size of clutch (number of ovules counted in each female), and mass of female and size of clutch for anuran species analyzed at Picinguaba, Ubatuba, state of São Paulo, Brazil from January 2000 to November 2002.

\begin{tabular}{|c|c|c|}
\hline Family/Species & $\begin{array}{l}\text { Log SVL }(\mathrm{mm}) \\
\text { vs. } \\
\text { Log size of clutch }\end{array}$ & $\begin{array}{l}\text { Log mass }(\mathrm{g}) \\
v s . \\
\text { Log size of clutch }\end{array}$ \\
\hline $\begin{array}{l}\text { BUFONIDAE } \\
\text { Rhinella ornata (Spix, 1824) } \\
\text { Dendrophryniscus brevipollicatus Jiménez de la Espada, } 1871\end{array}$ & $\begin{array}{l}\mathrm{R}^{2}=0.81 ; \mathrm{p}=0.02 ; \mathrm{n}=8 \\
\mathrm{y}=-4.11+4.14 \mathrm{x} \\
\mathrm{R}^{2}=0.58 ; \mathrm{p}=0.07 ; \mathrm{n}=6 \\
\mathrm{y}=-2.78+3.10 \mathrm{x}\end{array}$ & $\begin{array}{l}\mathrm{R}^{2}=0.60 ; \mathrm{p}=0.02 ; \mathrm{n}=8 \\
\mathrm{y}=2.02+1.14 \mathrm{x} \\
\mathrm{R}^{2}=0.34 ; \mathrm{p}=0.23 ; \mathrm{n}=6 \\
\mathrm{y}=1.46+0.77 x\end{array}$ \\
\hline $\begin{array}{l}\text { HYLIDAE } \\
\text { Aplastodiscus eugenioi (Carvalho-e-Silva \& Carvalho-e-Silva, 2005) }\end{array}$ & $\begin{array}{l}\mathrm{R}^{2}=0.26 ; \mathrm{p}=0.29 ; \mathrm{n}=6 \\
\mathrm{y}=-1.29+2.03 x\end{array}$ & $\begin{array}{l}\mathrm{R}^{2}=0.27 ; p=0.28 ; \mathrm{n}=6 \\
\mathrm{y}=1.62+0.56 \mathrm{x}\end{array}$ \\
\hline Hypsiboas albomarginatus (Spix, 1824) & $\begin{array}{l}\mathrm{R}^{2}=0.27 ; \mathrm{p}=0.15 ; \mathrm{n}=9 \\
\mathrm{y}=-0.32+1.95 x\end{array}$ & $\begin{array}{l}\mathrm{R}^{2}=0.01 ; \mathrm{p}=0.84 ; \mathrm{n}=7 \\
\mathrm{Y}=3+0.09 \mathrm{x}\end{array}$ \\
\hline Scinax angrensis (B. Lutz, 1973) & $\begin{array}{l}\mathrm{R}^{2}=0.02 ; \mathrm{p}=0.81 ; \mathrm{n}=5 \\
\mathrm{Y}=1.00+1.19 \mathrm{x}\end{array}$ & $\begin{array}{l}\mathrm{R}^{2}=0.01 ; \mathrm{p}=0.84 ; \mathrm{n}=5 \\
\mathrm{y}=2.69+0.38 \mathrm{x}\end{array}$ \\
\hline S. argyreornatus (Miranda-Ribeiro, 1926) & $\begin{array}{l}\mathrm{R}^{2}=0.33 ; \mathrm{p}=0.06 ; \mathrm{n}=11 \\
\mathrm{y}=11.39-7.33 \mathrm{x}\end{array}$ & $\begin{array}{l}\mathrm{R}^{2}=0.07 ; \mathrm{p}=0.43 ; \mathrm{n}=11 \\
\mathrm{y}=1.91-0.89 x\end{array}$ \\
\hline S. hayii (Barbour, 1909) & $\begin{array}{l}\mathrm{R}^{2}=0.69 ; \mathrm{p}=0.04 ; \mathrm{n}=6 \\
\mathrm{y}=-9.55+7.61 x\end{array}$ & $\begin{array}{l}\mathrm{R}^{2}=0.05 ; \mathrm{p}=0.66 ; \mathrm{n}=6 \\
\mathrm{y}=2.48+0.89 x\end{array}$ \\
\hline $\begin{array}{l}\text { HYLODIDAE } \\
\text { Hylodes asper (Müller, 1924) }\end{array}$ & $\begin{array}{l}\mathrm{R}^{2}=0.22 ; \mathrm{p}=0.42 ; \mathrm{n}=5 \\
\mathrm{y}=-17.22+11.63 \mathrm{x}\end{array}$ & $\begin{array}{l}\mathrm{R}^{2}=0.02 ; \mathrm{p}=0.84 ; \mathrm{n}=5 \\
\mathrm{y}=1.57+0.33 \mathrm{x}\end{array}$ \\
\hline $\begin{array}{l}\text { LEPTODACTYLIDAE } \\
\text { Leptodactylus marmoratus (Steindachner, 1867) }\end{array}$ & $\begin{array}{l}\mathrm{R}^{2}=0.01 ; \mathrm{p}=0.74 ; \mathrm{n}=19 \\
\mathrm{y}=2.21-1.15 \mathrm{x}\end{array}$ & $\begin{array}{l}\mathrm{R}^{2}=0.08 ; \mathrm{p}=0.22 ; \mathrm{n}=19 \\
\mathrm{y}=0.69+1.21 \mathrm{x}\end{array}$ \\
\hline $\begin{array}{l}\text { LEIUPERIDAE } \\
\text { Physalaemus atlanticus Haddad \& Sazima, } 2004\end{array}$ & $\begin{array}{l}\mathrm{R}^{2}=0.57 ; \mathrm{p}=0.01 ; \mathrm{n}=11 \\
\mathrm{y}=-2.87+3.51 x\end{array}$ & $\begin{array}{l}\mathrm{R}^{2}=0.49 ; \mathrm{p}=0.01 ; \mathrm{n}=11 \\
\mathrm{Y}=1.77+1.03\end{array}$ \\
\hline $\begin{array}{l}\text { MICROHYLIDAE } \\
\text { Chiasmocleis carvalhoi Cruz, Caramaschi \& Izecksohn, } 1997\end{array}$ & $\begin{array}{l}\mathrm{R}^{2}=0.19 ; \mathrm{p}=0.01 ; \mathrm{n}=29 \\
\mathrm{y}=-1.00+2.47 \mathrm{x}\end{array}$ & $\begin{array}{l}\mathrm{R}^{2}=0.31 ; \mathrm{p}=0.01 ; \mathrm{n}=29 \\
\mathrm{Y}=2.38+0.71 \mathrm{x}\end{array}$ \\
\hline Myersiella microps (Duméril \& Bibron, 1841) & $\begin{array}{l}\mathrm{R}^{2}=0.51 ; \mathrm{p}=0.11 ; \mathrm{n}=6 \\
\mathrm{y}=-1.01+1.61 x\end{array}$ & $\begin{array}{l}\mathrm{R}^{2}=0.14 ; \mathrm{p}=0.45 ; \mathrm{n}=6 \\
\mathrm{y}=1.14+0.41 \mathrm{x}\end{array}$ \\
\hline
\end{tabular}

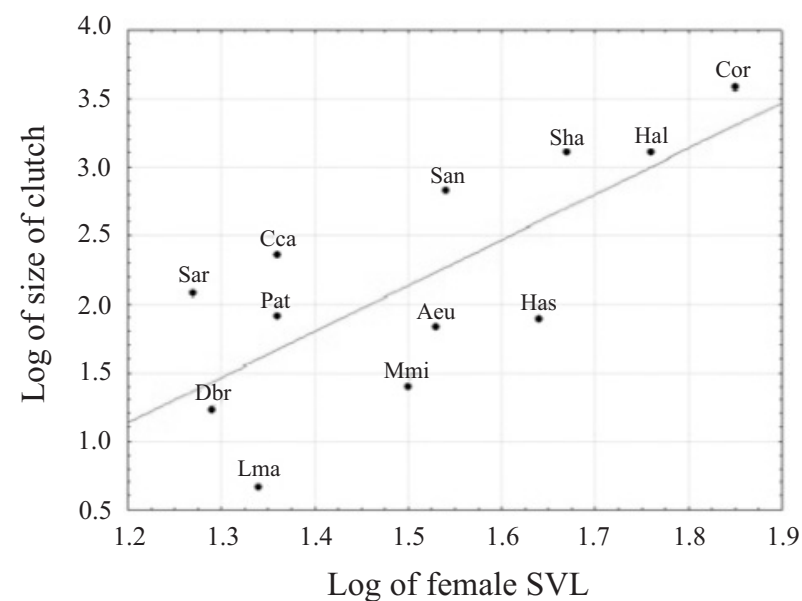

Figure 1. Relationship between mean log of female snout-vent length (SVL) and log of size of clutch for 12 species of anuran amphibians from Picinguaba, Ubatuba, state of São Paulo, Brazil $(\operatorname{logy}=-2.87+3.34 \operatorname{logx})$ (Aeu, Aplastodiscus eugenioi; Cca, Chiasmocleis carvalhoi; Cor, Rhinella ornata; Dbr, Dendrophryniscus brevipollicatus; Hal, Hypsiboas albomarginatus; Has, Hylodes asper; Lma, Leptodactylus marmoratus; Mmi, Myersiella microps; Pat, Physalaemus atlanticus; San, Scinax angrensis; Sar, Scinax argyreornatus; Sha, Scinax hayii).

\section{DISCUSSION}

Anuran reproductive modes have evolved along different pathways due to selective pressures such as environmental conditions, predation and interespecific competition (Duellman, 1989a). Reproductive mode 1 (eggs and tadpoles in lentic waters) is a generalized mode

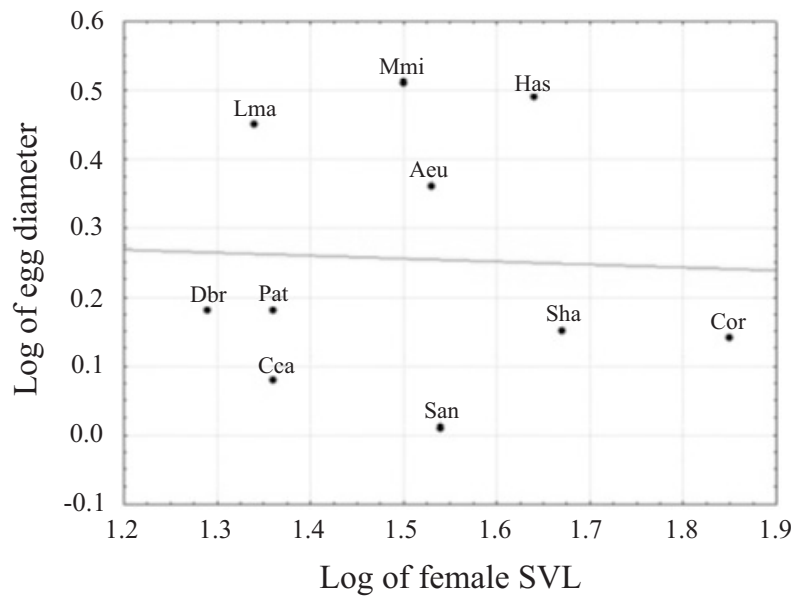

Figure 2. Relationship between mean log of female snout-vent length (SVL) and log of egg diameter for 12 species of anuran amphibians from Picinguaba, Ubatuba, state of São Paulo, Brazil $(\log \mathrm{y}=0.32-0.04 \log \mathrm{x})($ Aeu, Aplastodiscus eugenioi; Cca Chiasmocleis carvalhoi; Cor, Rhinella ornata; Dbr, Dendrophryniscus brevipollicatus; Has, Hylodes asper; Lma Leptodactylus marmoratus; Mmi, Myersiella microps; Pat, Physalaemus atlanticus; San, Scinax angrensis; Sha, Scinax hayii).

(e.g. Crump, 1974; Duellman, 1978; Duellman \& Trueb, 1986) and was the most common mode amongst the species of anurans observed at Picinguaba. Another 12 reproductive modes were recorded for the study area, confirming the high diversity of reproductive modes found in the Atlantic rainforest (HADDAD, 1998; HADDAD $\&$ PRADO, 2005). Considering that there are 39 recognized 
anuran reproductive modes in the world (HADDAD \& PRADO, 2005), 33.3\% are found among the anurans of Picinguaba. Of the 27 recognized modes for the Atlantic rainforest (HADDAD, 1998; HADDAD \& SAWAYA, 2000), $48.1 \%$ are found in Picinguaba. Moreover, of the nine reproductive modes recognized for hylids in the Atlantic rainforest (HADDAD \& SAWAYA, 2000; HADDAD \& PRADO, 2005; excluding the representatives of Amphignathodontidae, see Frost et al., 2006), six or $66.7 \%$ occur in Picinguaba.

The diversity of reproductive modes observed in the Atlantic rainforest is the result of the successful use by anurans of the diverse and humid microhabitats present in this biome (HADDAD \& SAWAYA, 2000) and indicates spatial partitioning of the environment. Different reproductive modes allow species to use habitat resources in different ways (SAlthe \& Duellman, 1973). The great number of habitats and microhabitats used for oviposition and larval development concurs with the idea that the efficient use of the available reproductive resources allows a great number of species to share the same environment (CRUMP, 1974, 1982; DuEllMAN, 1989b; AichINGER, 1992).

The high humidity, temperature and rainfall of tropical forests have possibly influenced the evolution of alternative reproductive modes (HöDL, 1990). The availability of water bodies in the Atlantic rainforest, even during the dry period, is due to sporadic rainfalls. Of the 40 species of anurans studied, 26 deposit eggs directly in the water and are totally dependent on water for all the stages of larval development. Up to 12 species of anurans were recorded calling syntopically in the same night from the same semipermanent pond.

Reproductive modes sustain a certain allegiance to the environment used by the species (LYNCH, 1979; Duellman, 1982, 1988). The species that used lentic waters, mainly in mode 1 , were found in a larger number of reproductive sites and were common in the studied assemblage. Rhinella ornata was found both in lentic and lotic waters (modes 1 and 2) and was the most generalist species. Physalaemus atlanticus showed two reproductive modes: 11 and 28 , and was found reproducing in the leaf litter or in temporary ponds and also occupied diverse environments inside the forest. All other reproductive modes belonged to species that had very specific reproductive sites.

Reproductive modes 2, 3, 5, 8, 19, 23, 25, and 28 are typical of forest environments and make up $61.5 \%$ of the observed modes. Reproductive modes 1, 4, and 11 occur both in open and forested areas and are common in species with environmental plasticity. Examples are Hypsiboas faber (mode 1 and 4), Dendropsophus minutus (mode 1), and Leptodactylus latrans (mode 11). Modes 24 and 32 are also considered to occur in open or forested areas (HADDAD \& PRADO, 2005), but in Picinguaba they were characteristic of two species associated to the forest: $D$. berthalutzae and L. marmoratus, respectively.

Excluding the reproductive modes associated strictly with ponds or streams, according to HADDAD \& PRADO (2005) the other more specialized reproductive modes in the Atlantic rainforest can be divided into two large groups: modes associated with vegetation (in the present study modes 8,24 , and 25) and modes associates with the floor (in the present study modes 4, 5, 19, 23, and $32)$. Reproductive modes associated with vegetation appeared in Bufonidae (mode 8), Centrolenidae (mode 25), and Hylidae (modes 24 and 25). These last two families use the vegetation mainly for reproduction. Five reproductive modes associated with the floor were found in six families of anurans in Picinguaba: Brachycephalidae (mode 23, only reproductive mode observed, according to the proper diagnosis for the family as reorganized by FROst et al., 2006), Cycloramphidae (mode 19), Hylidae (modes 4 and 5), Leptodactylidae (mode 32), and Microhylidae (mode 23). The only family with reproductive modes associated both with the floor and the vegetation was Hylidae. As suggested by HADDAD \& PRADO (2005), the differences in the ratio of reproductive modes associated with the floor and the vegetation in different taxa reflect the general patterns of adaptation toward habitat occupation, in this case, the structure of the studied taxocenose. This characterization of the groups and their reproductive specializations in a heterogeneous environment such as the Atlantic rainforest show the history of occupation of the region by the different phylogenetic groups of anurans.

An interesting characteristic of species reproduction is the oviposition behavior that can vary within a single reproductive mode. This differentiation was observed mainly in mode 1 , where $R$. ornata, $T$. mesophaeus, C. carvalhoi and I. langsdorffii deposited their eggs in different oviposicional modes (sensu AltiG $\&$ MCDIARMID, 2007). This behavioral variation is part of the life history of each species and can be related to the search for the best site for oviposition, considering the intensity of light and/or concentration of oxygen for the fast development of the embryos. Some sites contained a lot of vegetation and the eggs seemed to be protected from direct sunlight. Clutches that were deposited glued to aquatic vegetation could benefit from the protection accorded to these plants, which may avoid direct sunlight and the plants could also act as anchors for the spawn. The spawn of the species in general seem to be placed in the best available site at the moment of oviposition, since many pairs deposited their eggs in the same night in the limited space of small breeding sites.

Communities formed by species with small SVLs were also found in other studies of amphibians (CRUMP, 1974; Aichinger, 1992). According to Crump (1974), most of the populations of amphibians have a small optimum body size, with the exception of amphibians that have evolved from medium-sized ancestors. The size of the reproductive phase may also be related to a certain dependence on water. In the case of Picinguaba, $R$. ornata and $M$. alipioi are large species with mode 1 , and $L$. latrans, the largest species found in the area, has mode 11. Reproductive mode 1 is characterized by a large number of small aquatic eggs. In mode 11, a foam nest is placed in a pond and the exotrophic tadpoles develop in lentic waters. As concluded by POMBAL \& HADDAD (2005), while studying a taxocenose of amphibians in Paranapiacaba, São Paulo, Brazil, it is likely that problems of gaseous exchange between large eggs and the environment are the limiting factor for large-bodied species to have evolved complete terrestrial development.

Sexual dimorphism, with larger females than males, is common in amphibians (SHINe, 1979). The size of the female can affect the size and number of eggs (KAPLAN \& SALTHE, 1979) and the positive correlation between SVL and number of eggs in the species from Picinguaba show this fact clearly. Thus, the egg number that each female is capable of producing increases with the size of the body 
(JoRGENSEN, 1992), which explains the larger size of females (SHINE, 1979).

The size of the body explained between $57 \%$ and $81 \%$ of the variation in the size of the clutch, but only three species had statistically significant results. Egg production can vary considerably between females of the same population (LEMCKERT \& SHINE, 1993; PRADO \& HADDAD, 2005), or may be regulated by climatic conditions (CRUMP, 1974). In a study in the Brazilian Pantanal that measured these variables in females, a positive correlation was found in most of the studied species (PRADO \& HADDAD, 2005). However, in the Atlantic rainforest, the species can reproduce practically throughout the whole year, different from what occurs in the Pantanal where there are distinct periods of drought and rainfall. It is possible that females of anurans in Picinguaba deposit fewer eggs per clutch, but reproduce many times during the year. However, in the present study, the number of times a species can reproduce per year was not assessed (e.g. SAlthe \& DuEllman, 1973) nor were environmental factors such as food and temperature specific for each species (JORGENSEN, 1992). Another limitation was the number of females analyzed for each species, which was low for statistical analyses.

The number of eggs was correlated with the SVL of the females, confirming a trend already observed for other populations of amphibians (SALTHE \& DuEllman, 1973; Crump, 1974; Duellman, 1989b; Prado \& Haddad, 2005). Aquatic reproductive modes had a larger number of eggs than terrestrial or arboreal modes. However, the diameter of the egg was not statistically correlated with the size of the female. Other factors may be influencing the size of eggs, such as the age of the female, or the annual reproductive effort (e.g. CunNington \& BROoKs, 2000). The size of the egg may also depend on the environment and may be related to abundance of food, size of the female, and temperature of the female during vitelogenesis (KAPLAN, 1987). The species with bigger eggs were those with specialized reproductive modes: $M$. microps has direct development, L. marmoratus deposits eggs in foam nests inside burrows, A. eugenioi deposits its eggs in an underground chamber constructed by the male, $H$. asper deposits its eggs in a submerged burrow in running water, and $P$. atlanticus deposits eggs in a foam nest, either in the leaf litter or in water in temporary ponds. The only species with a specialized reproductive mode that had an egg diameter close to that expected for non specialized reproductive modes (mode 1 ) was $D$. brevipolicatus, which deposits eggs in the water of bromeliads and was the smallest species analyzed. An average of $18.5 \mathrm{eggs}$ of $1.59 \mathrm{~mm}$ of diameter was deposited by females with an average SVL of $19.72 \mathrm{~mm}$.

The diameter of the egg and the number of eggs are important parameters concerning size-fecundity relationships (LANG, 1995). Some studies found a negative relationship between the diameter of the egg and the number of eggs per clutch (Salthe, 1969; Salthe \& Duellman, 1973; CRumP, 1974; Duellman, 1989b), as was also found in the species of anurans in Picinguaba. This means that the smaller the ovule produced by a female, the greater will be the number of ovules it will be able to produce and carry (LANG, 1995).

In the evolutionary sequence from aquatic to terrestrial eggs there is a reduction in the number of eggs and an increase in the size of the eggs (MARTIN, 1967; Pombal, 1999). The larger number of eggs produced for species with aquatic clutches when compared to terrestrial species, also recorded for the anurans in Picinguaba, agrees with this idea. This is due to the fact that an increase in the size of eggs implies a reduction in the number of eggs per clutch (POMBAL \& HADDAD, 2005). Bigger eggs and embryos consume more oxygen (Kuramoto, 1975; 1978) besides having slower development (Salthe \& Duellman, 1973; Pombal \& HADDAD, 2005). According to this idea, the more terrestrial the development, culminating in direct development, the bigger is the relative size of the egg. For example, Microhylidae: $C$. carvalhoi, with aquatic development (mode 1), has eggs that are $5.3 \%$ the average SVL of the female and there is an average of 236 eggs per clutch, whilst $M$. microps, with direct development (mode 23), has eggs that are $10.2 \%$ the average SVL of the female and there is an average of 25 eggs per clutch.

Fecundity, measured in number of eggs, was significantly correlated with the SVL of the female in species with non specialized reproductive modes, but not in species with specialized reproductive modes. The diameter of the egg relative to the SVL of the female was not significant for any type of reproductive mode. These parameters may be related more to environmental variables or even to the low sample than to the physiological and ecological processes of the studied populations. Quantitative studies are important for understanding community ecology; however, fecundity measured by number of eggs or size of eggs does not imply in greater or lower reproductive success, in terms of descendants left for the next generation. For each reproductive mode, there may be an optimum egg diameter or number of hatchlings (e.g. Nussbaum, 1987; Semlitsch \& GibBons, 1990). Efficient reproductive modes are an important aspect for the survival of the populations and, potentially, the species (Duellman, 1985).

Acknowledgments. We thank C. P. A. Prado, L. Lugli, M. Barbosa, F. Toledo, P. C. A. Garcia and L. O. M. Giasson for help in the field and A. Heursel Baldisseri for the English version; Instituto Florestal, Parque Estadual da Serra do Mar, Núcleo Picinguaba, for permitting access to the park and for logistic support; Fundação O Boticário de Proteção a Natureza and McArthur Foundation for financial support of the project "Amphibian Decline in the Atlantic Forest”; CNPq, CAPES, and FAPESP for financial support.

\section{REFERENCES}

Aichinger, M. 1992. Fecundity and breeding sites of an anuran community in a seasonal tropical environment. Studies on Neotropical Fauna and Environment 27:9-18.

Altig, R. \& McDiarmid, R. W. 2007. Diversity, morphology, and evolution of egg and clutch structure in amphibians. Herpetological Monographs 21:1-32.

Bertoluci, J. 1998. Annual patterns of breeding activity in Atlantic rainforest anurans. Journal of Herpetology 32:607-611.

Bertoluci, J. \& Rodrigues, M. T. 2002. Seasonal patterns of breeding activity of Atlantic rainforest anurans at Boracéia, Southeastern Brazil. Amphibia-Reptilia 23:161-167.

Boquimpani-Freitas, L.; Rocha, C. F. D. \& Sluys, M. V. 2002. Ecology of the horned leaf-frog Proceratophrys appendiculata (Leptodactylidae), in an insular Atlantic rain-forest area of southeastern Brazil. Journal of Herpetology 36:318-322.

Caldwell, J. P. 1992. Diversity of reproductive modes in anurans: facultative nest construction in gladiator frogs. In: HamLetT, W. C. ed. Reproductive biology of South American vertebrates. New York, Springer-Verlag. p.85-97.

Carvalho, A. L. 1949. Notas sobre os hábitos de Dendrophryniscus brevipollicatus Espada (Amphibia, Anura). Revista Brasileira de Biologia 9:223-227.

Carvalho-e-Silva, S. P.; Pinto, A. L. C. \& Carvalho-e-Silva, A. M. 
P. T. 2002. Aspectos da reprodução, da vocalização e da larva de Phrynohyas mesophaea Hensel (Amphibia, Anura, Hylidae). Aquarium 15:19-24.

Crump, M. L. 1974. Reproductive strategies in a tropical anuran community. Miscellaneous Publication, University of Kansas Museum of Natural History 61:1-68.

1982. Amphibian reproductive ecology on the community level. In: ScOTT JR., N. J. ed. Herpetological communities. Washington, Wildlife Research Report. p.21-36.

Cunnington, D. C. \& Brooks, R. J. 2000. Optimal egg size theory: does predation by fish affect egg size in Ambystoma maculatum? Journal of Herpetology 34:46-53.

Duellman, W. E. 1978. The biology of an equatorial herpetofauna in Amazonian Ecuador. Miscellaneous Publication, University of Kansas Museum of Natural History 5:1-352. 1982. Quaternary climatic-ecological fluctuations in the lowland tropics: frogs and forest. In: PrAncE, J. T. ed. Biological diversification in the tropics. New York, Columbia University. p.389-402.

1985. Reproductive modes in anuran amphibians: Phylogenetic significance of adaptive strategies. South African Journal of Science 81:174-178.

1988. Pattern of species diversity in anuran amphibians in the American Tropics. Annals of the Missouri Botanical Garden 75:79-104.

1989a. Alternative life-history styles in anuran amphibians: evolutionary and ecological implications. In: BRUTON, M. H. ed. Alternative life history of animals. Dordrecht, Kluwer Academic. p.101-126.

. 1989b. Tropical herpetofaunal communities: Patterns of community structure in Neotropical rainforests. In: HARMELINVievien, M. L. \& Bourliére, F. eds. Ecological studies. Vertebrates in complex tropical systems. New York, Springer. v.69, p.61-68.

Duellman, W. E. \& Thomas, R. 1996. Anuran amphibians from a seasonally dry forest in southeastern Peru and comparisons of the anuran among sites in the upper Amazon Basin. Occasional Papers Museum of Natural History, University of Kansas 180:1-34.

Duellman, W. E. \& Trueb, L. 1986. Biology of amphibians. New York, McGraw-Hill. 670p.

Frost, D. R. 2009. Amphibian species of the world: an online reference, v. 5.3. New York, American Museum of Natural History. Avaliable at: <http://research.amnh.org/ herpetology/amphibia/>. Access on: 12.02.2009.

Frost, D. R.; Grant, T.; Faivovich, J.; Bain, R. H.; HaAs, A.; Haddad, C. F. B.; De Sá, R. O.; Channing, A.; Wilkinson, M.; Donnellan, S. C.; RaXworthy, C. J.; Campbell, J. A.; Blotto, B. L.; Moler, P.; Drewes, R. C.; Nussbaum, R. A.; Lynch, J. D.; Green, D. M. \& Wheeler, W. C. 2006. The amphibian tree of life. Bulletin of the American Museum of Natural History 297:1-371.

Haddad, C. F. B. 1998. Biodiversidade dos anfíbios no Estado de São Paulo. In: CAstro, R. M. ed. Biodiversidade do Estado de São Paulo, Brasil: síntese do conhecimento ao final do século XX. São Paulo, FAPESP. p.15-26.

Haddad, C. F. B. \& GiarettA, A. A. 1999. Visual and acoustic communication in the Brazilian torrent frog, Hylodes asper (Anura: Leptodactylidae). Herpetologica 55:324-333.

Haddad, C. F. B. \& Prado, C. P. A. 2005. Reproductive modes in frogs and their unexpected diversity in the Atlantic forest in Brazil. BioScience 55:207-217.

HadDAD, C. F. B. \& SAwAya, R. 2000. Reproductive modes of Atlantic forest hylid frogs: a general overview and description of a new mode. Biotropica 32:862-871.

Haddad, C. F. B. \& Sazima, I. 2004. A new species of Physalaemus (Amphibia; Leptodactylidae) from the Atlantic forest in southeastern Brazil. Zootaxa 479:1-12.

Hartmann, M. T.; Hartmann, P. A. \& Haddad, C. F. B. 2004. Visual signaling and reproductive biology in a nocturnal treefrog, genus Hyla (Anura: Hylidae). Amphibia-Reptilia 25:395-406.

Heyer, W. R.; Rand, A. S.; Cruz, C. A. G.; Peixoto, O. L. \& Nelson, C. E. 1990. Frogs of Boracéia. Arquivos de Zoologia 31:231-410.

HöDL, W. 1990. Reproductive diversity in Amazonian lowland frogs. In: Hanke, W. ed. Biology and Physiology of Amphibians. Stuttgart, Gustav Fischer Verlag. p.41-60.
IzECKSOHN, E. \& JIM, J. 1971. Observações sobre o desenvolvimento de Myersiella subnigra (Miranda-Ribeiro). Arquivos do Museu Nacional 54:69-73.

Jorgensen, C. B. 1992. Growth and reproduction. In: Feder, M. E. \& Burgaren, W. W. eds. Environmental physiology of the amphibians. Chicago, University of Chicago. p.439-466.

Kaplan, R. H. 1987 . Developmental plasticity and maternal effects of reproductive characteristics in the frog, Bombina orientalis. Oecologia 71:273-279.

Kaplan, R. H. \& Salthe, S. N. 1979. The allometry of reproduction: an empirical view in salamanders. American Naturalist 113:671-689.

Kuramoto, M. 1975. Adaptative significance in oxygen consumption of frog embryos in relation to the environmental temperatures. Comparative Biochemistry and Physiology 52:59-62.

1978. Correlations of quantitative parameters of fecundity in amphibians. Evolution 32:287-269.

LANG, C. 1995. Size-fecundity relationships among stream-breeding hylid frogs. Herpetological Natural History 3:193-197.

Lemckert, F. L. \& Shine, R. 1993. Costs of reproduction in a population of the frog Crinia signifera (Anura: Myobatrachidae) from southeastern Australia. Journal of Herpetology 27:420-425.

LYNCH, J. D. 1979. The amphibians of the lowland tropical forest. In: Duellman, W. E. ed. The South American Herpetofauna: its origin, evolution, and dispersal. Monographs of the Museum of Natural History, Lawrence, University of Kansas, 7. p.189-215.

Martin, A. A. 1967. Australian anuran life histories: some evolutionary and ecological aspects. In: Weatherley, A. S. ed. Australian inland waters and their fauna. Canberra, Canberra University. p.175-191.

Martins, M.; Pombal, J. P. \& Haddad, C. F. B. 1998. Escalated aggressive behaviour and facultative parental care in the nest building gladiator frog, Hyla faber. Amphibia-Reptilia 19:6573 .

Nussbaum, R. A. 1987. Parental care and egg size in salamanders, an examination of the safe harbour hypothesis. Researches on Population Ecology 29:27-44.

Pombal, J. P. 1999. Oviposição e desenvolvimento de Brachycephalus ephippium (Spix) (Anura, Brachycephalidae). Revista Brasileira de Zoologia 16:967-976.

Pombal, J. P. \& Haddad, C. F. B. 2005. Estratégia e modos reprodutivos de anuros (Amphibia) em uma poça permanente na Serra de Paranapiacaba, Sudeste do Brasil. Papéis Avulsos de Zoologia 45:201-213.

Pombal, J. P.; Sazima, I. \& Haddad, C. F. B. 1994. Breeding behavior of the pumpkin toadlet, Brachycephalus ephippium (Brachycephalidae). Journal of Herpetology 28:516-519.

Prado, C. P. A. \& Haddad, C. F. B. 2005. Size-fecundity relationships and reproductive investment in female frogs in the Pantanal, south-western Brazil. Herpetological Journal 15:181-189.

Prado, C. P. A.; Uetanabaro, M. \& Haddad, C. F. B. 2002. Description of a new reproductive mode in Leptodactylus (Anura, Leptodactylidae), with a review of the reproductive specialization toward terrestriality in the genus. Copeia 2002:1128-1133.

SAlthe, S. N. 1969. Reproductive modes and the number and sizes of ova in urodeles. American Midland Naturalist 81:467-490.

Salthe, S. N. \& Duellman, W. E. 1973. Quantitative constraints associated with reproductive mode in anurans. In: VIAL, J. L. ed. Evolutionary biology of the anurans. Contemporary research on major problems. Columbia, University of Missouri. p.229-249.

Semlitsch, R. D. \& Gibbons, J. W. 1990. Effects of egg size on success of larval salamanders in complex aquatic environments. Ecology 71:1789-1795.

Shine, R. 1979. Sexual selection and sexual dimorphism in the Amphibia. Copeia 1979:297-306.

Veloso, H. P.; Rangel Filho, A. L. R. \& Lima, J. C. A. 1991. Classificação da vegetação brasileira, adaptada a um sistema universal. Rio de Janeiro, IBGE. 123p.

Wells, K. D. 1977. The social behaviour of anuran amphibians. Animal Behaviour 25:666-693. 